\title{
The spheroplastics destruction mechanisms under hydrostatic compression
}

\author{
Robert Turusov ${ }^{1,3, *}$, Vitaliy Solodilov ${ }^{1}$, Alexsey Kireynov ${ }^{2}$, Roman Korokhin ${ }^{1}$, Alexander \\ Kuperman ${ }^{1}$ \\ ${ }^{1}$ Semenov institute of chemical physics Russian academy of sciences, ul. Kosygyna, 4, 119991, \\ Moscow, Russia \\ ${ }^{2}$ Bauman Moscow state technical university, ul. Baumanskaya 2-ya, 5/1, 105005, Moscow, Russia \\ ${ }^{3}$ Moscow State University of Civil Engineering, Yaroslavskoe Shosse, 26, Moscow, Russia
}

\begin{abstract}
This paper is devoted to hydrostatic compression testing of glass microspheres and spheroplastics (syntactic foam) based on them. Despite a significant share of the void, for example, with a volume fraction of glass microspheres in the syntactic foam of 0.5 , the share of the void will be equal to 0.447 . The opposition to hydrostatic compression of this material is surprising. The authors consider that the whole point here is to determine the signs and criteria of destruction of these materials. That is why tests were conducted to determine the strength of spheroplastics and a set of glass microspheres with modeling in the composition of an aqueous emulsion.
\end{abstract}

\section{Introduction}

One of the composites, combining a very low density with high stiffness and strength, and which are increasingly used in various branches of human activity, are syntactic foams. Fillers in them are thin-walled microspheres from different materials. The main application of such materials is the fillers for various products. Special attention is paid to syntacticsin the creation of deep-sea apparatus. In this regard, the criteria for the destruction of such materials under compression, especially under hydrostatic one, are particularly relevant. In [1-9], syntactic composites under uniaxial compression and hydrostatic compression are investigated. Most of these studies do not show a complete picture of the destruction of syntactic foam, the strength criteria have not been determined yet. The main purpose of this work is to determine the criteria of destruction of syntactic composites under hydrostatic compression both experimentally and with the help of mathematical modeling.

\section{Materials and methods}

We used glass microspheres MS-VP-A9 (JSC "NPO Stekloplastik"). Earlier in the work $[1,2]$, we conducted research to determine the geometric and physical parameters of microspheres, which are presented in Fig.1.

\footnotetext{
*Corresponding author : rob-turusov@yandex.ru
} 
Syntactic composites were obtained by pressing plates. The binder was an epoxy resin cured with alkofen at room temperature for 24 hours. Cylindrical samples with the diameter of $10 \mathrm{~mm}$ and the height of $20 \mathrm{~mm}$ were made by turning treatment. The volume content of glass microspheres in the syntactic composite ranged from 30 to 90 vol. \%. For each type of material, we produced three samples for hydrostatic compression testing and two samples for determining the front of damage propagation during fracture. In addition, samples of glass microspheres were tested.

Hydrostatic compression of glass spheres and syntactic composites on their basis was performed in the equipment, developed by us, described earlier in [2]. The hydraulic cylinder was loaded with the speed of the upper clamp of the testing machine of $1 \mathrm{~mm} / \mathrm{min}$. During loading, a diagram of the force dependence $F$ on time $t$ was recorded.

(a)

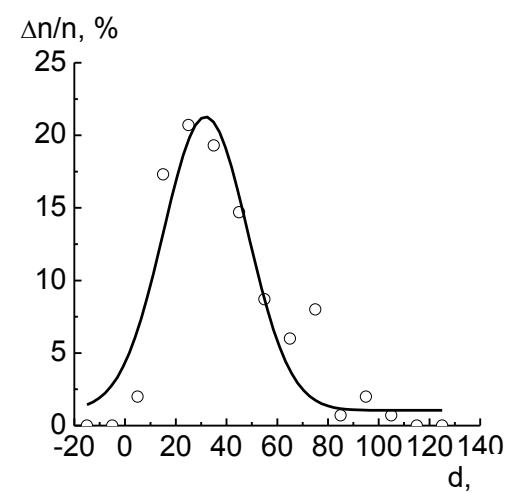

(b)

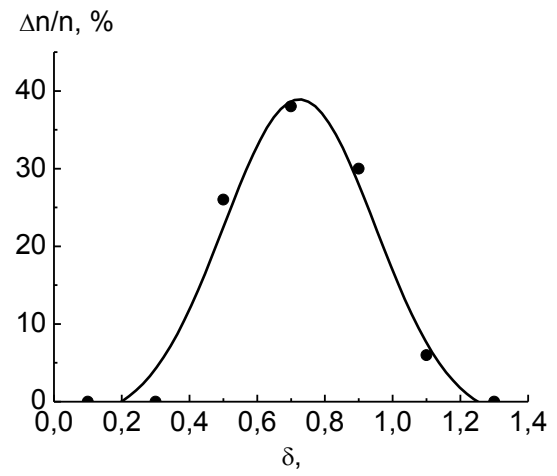

Fig. 1. Diameter distribution curve (a) $\left(d_{\text {mid }}=39,8 \mathrm{mkm} ; 0 \leq d_{c p} \leq 103 \mathrm{mkm} ; \sqrt{ } D_{d} / d=0,53 ; \Delta=0,4\right.$ $\mathrm{mkm}$; error $-0,9 \%)$ and the distribution curve for the thickness of the walls of the glass microspheres (б) $\left(\delta_{\text {mid }}=0,75 \mathrm{mkm} ; 0,22 \leq \delta_{\text {mid }} \leq 1,27 ; \sqrt{ } \mathrm{D}_{\delta} / \delta=0,235 ; \Delta=0,025\right.$; error $\left.-3,3 \%\right)$ [2] .

To visualize the area of destruction, samples of syntactics were kept in a hydraulic cylinder with colored water at a pressure of $250 \mathrm{MPa}$ for 10 minutes. Control samples were also kept in colored water with dye at atmospheric pressure. The stained samples were examined visually after test. The depth $l$ of the dye penetration front with respect to the radius $\mathrm{R}_{\mathrm{S}}$ of the sample was estimated.

\section{Results and discussion}

\subsection{Experiment}

Fig. 2 shows the loading diagrams of syntactic composites and microspheres in a hydraulic cylinder, as well as the nature of pressure growth in water without samples. The diagrams show that the pressure in the hydraulic cylinder increases almost linearly until the beginning of the destruction of materials. The moment of fracture is characterized by an inflection in the diagram P-t. Probably, this inflection appears due to the destruction of microspheres. As a result, all the diagrams with samples demonstrate the nonlinearity of pressure changes depending on time. Under further loading, the diagrams are linear. It should be noted that the inflection in the diagram $\mathrm{P}-\mathrm{t}$ characterizes the destruction of the material. From the loading diagrams, the pressures of the beginning, middle and end of the destruction of syntactic composites were determined. The results of these calculations are shown in Fig. 3. 


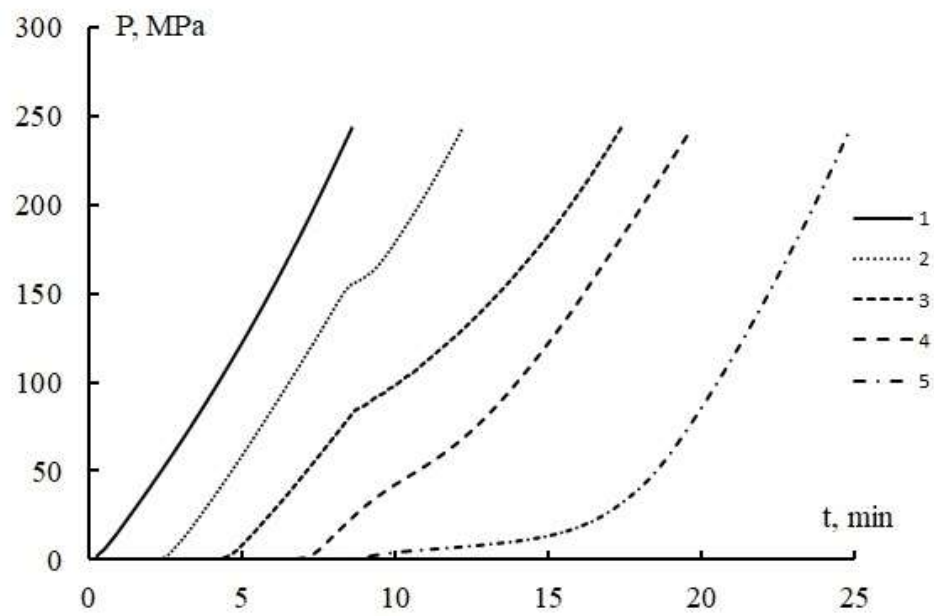

Fig. 2. Loading diagrams of the hydraulic cylinder without samples (1), samples containing 30 vol.\% (2) 60 vol. \% (3) 90 vol. \% (4) of microspheres and pure glass microspheres (5).

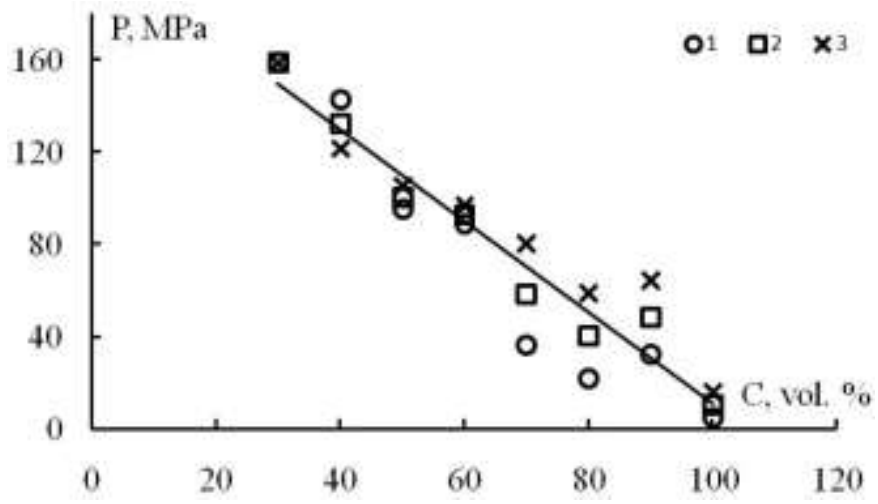

Fig. 3. The dependence of the strength of syntactic composites on the volume content of hollow glass microspheres C. Strengths corresponding to the beginning (1), the middle (2) and the end (3) of the destruction process.

Fig. 3 shows that the strength of syntactic composites decreases linearly with increasing of the volume content of glass microspheres. The pressure values characterizing the beginning, middle and end of the destruction of the samples in the range of the volume content of glass microspheres of 30-60 vol. \% are close to each other. At concentrations above 60 vol. \% the process of destruction of the material is more stretched over a range of pressures: the pressure corresponding to the beginning of the destruction is two times lower than the pressure at which the destruction ends. Probably, this difference is due to the variability strengthof the glass spheres (Fig. 1).

Fig. 4 shows that for samples held without pressure, the dye penetrated only into the thin outer layer of the samples. The penetration depth in this case does not depend on the concentration of the glass microspheres. When the samples of syntactics are held under pressure, the depth of penetration of the dye depends on the volume content of the microspheres in the sample. At the same time, the higher the concentration of glass microspheres, the deeper the sample is painted. It can be assumed that the penetration depth of the dye corresponds to the depth of destruction of the sample material during compression. 


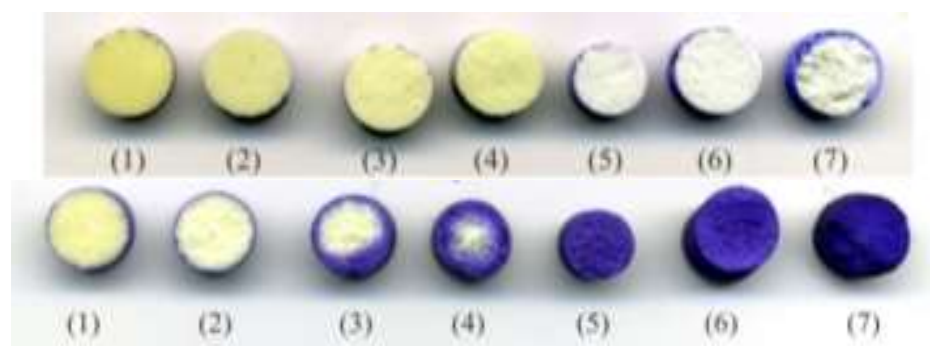

Fig. 4. Samples of syntactic composites after exposure without external pressure (a) and after exposure under pressure (b). Volume content of glass microspheres: (1) - 30 vol. \%, (2) - 40 vol. \%, (3) -50 vol. $\%$, (4) - 60 vol. \%, (5) - 70 vol. \%, (6) - 80 vol. $\%$, (7) - 90 vol. $\%$.

Fig. 5 shows the process of dye penetration, characterizing the propagation of the destruction front. It can be seen that in the range of the volume content of microspheres $30-$ $40 \mathrm{vol} . \%$ fracture depth is insignificant and is less than $10 \%$ of the sample radius. When the content of microspheres in the syntactic composite is from 40 to 70 vol. \%, a linear increase in the fracture front is observed with an increase in the filler concentration. At higher concentrations, the ratio $l$ to the radius $\mathrm{R}_{\mathrm{S}}$ of the sample is 1 , i.e. the fracture front has reached the center of the sample.

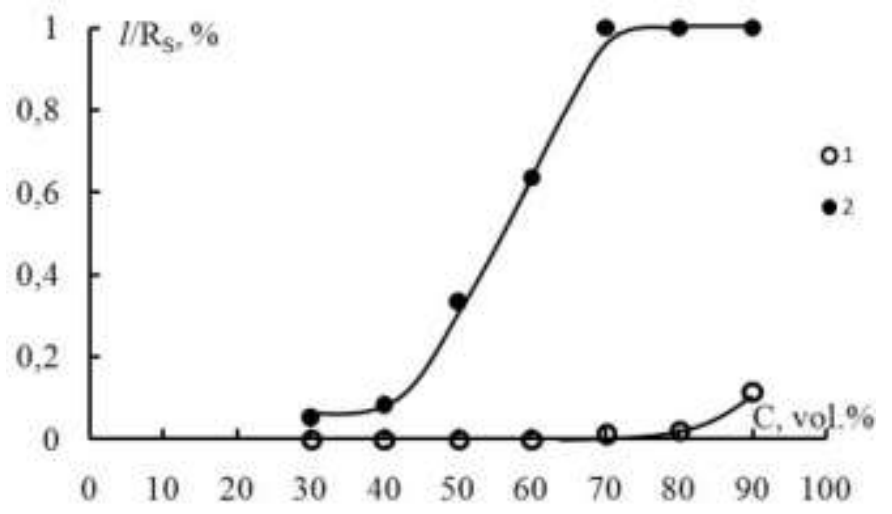

Fig. 5. Dependence of the depth of the fracture front $l$ to the radius $\mathrm{R}_{\mathrm{S}}$ of the sample on the content of glass microspheres in the syntactic composite.

\subsection{Modeling of hydrostatic compression of glass microspheres in the composition of aqueous suspension}

Fig. 2 and 3 demonstrate that the fracture process is strongly stretched in the pressure range. Variation in the strength of glass microspheres due to the distribution of their geometric parameters (Fig. 1) does not fully explain this fact. To answer on the question why this happens, the authors conducted mathematical modeling and compression tests of the aqueous suspension of the glass microspheres.

In the composition of syntactic foam, glass microspheres have a large fraction of the void $r_{1}^{3} / R^{3}=0.895$. For example, when the volume fraction of the glass microspheres in syntactic foam is 0.5 , the fraction of the void is 0.447 . The distribution of geometric parameters in these tests, as in Fig. 1, can also play a significant role. 
Here we are talking about modeling the hydrostatic compression of a suspension of the glass microspheres - water in two different modes:

1. Compression with a constant rate of pressure growth $p=p_{0}+q t$;

2. Compression with a constant rate of decrease in the volume of the suspension $V=V_{0}+k t$.

Notations: $t$ - time, $k$ and $q$ - rate of change of volume and pressure, respectively.

\subsubsection{Glass sphere under external pressure}

We are primarily interested in displacement of the points of the outer radius when the sphere is compressed $u(R)$. It is definitely related to the circumferential deformation $\varepsilon_{\varphi}=\varepsilon_{\theta}$. From Hooke's law, equilibrium equations and Cauchy relations:

$$
\varepsilon_{\varphi}=\frac{1}{E}\left[\sigma_{\varphi}-\mu\left(\sigma_{r}+\sigma_{\theta}\right)\right], \varepsilon_{\varphi}=\frac{u}{r}
$$

We obtain for displacement on the outer surface of the sphere:

$$
u(R)=\varepsilon_{\varphi}(R) R=p \frac{R}{2 E\left(R^{3}-r_{1}^{3}\right)}\left[2 R^{3}(1-2 \mu)+r_{1}^{3}(1+\mu)\right]
$$

The volume change in the compression of one microsphere is calculated by the formula:

$$
\Delta V_{1 s p h} \approx 4 \pi R^{2} u \approx 6 \frac{p}{E} \frac{R^{4}}{\delta}(1-\mu) ; \delta=R-r_{1}<<r_{1}
$$

When a single microsphere is fractured, the volume is released:

$$
v_{r e l}=\frac{4}{3} \pi r_{1}^{3} \approx \frac{4}{3} \pi R^{3}\left(1-3 \frac{\delta}{R}\right)
$$

\subsubsection{Compression of suspension - microspheres in water}

There is a cylindrical capsule with diameter $\mathrm{b}$ and height $\mathrm{L}$. Its volume is equal to $V_{k}=\pi b^{2} L$ . The volume content of microspheres in the water is $\mathrm{n}\left(1 / \mathrm{m}^{3}\right)$.

If we consider the radius of the piston $b$ unchanged, the total change in the volume of the capsule during compression of the suspension is:

$$
\Delta V=\pi b^{2} \Delta L=\pi b^{2} L\left[6 n \frac{p}{E}(1-\mu) \frac{R^{4}}{\delta}+p\left(1-v_{n}\right) \beta_{\text {water }}\right]
$$

Geometric parameters (Fig.1) microspheres are contained in formulae for stresses and strains. These values are important strength characteristics of the microspheres. Formulae for strength will be significantly complicated if we take into account the spread of geometric parameters. At the same time, the actual picture of the destruction of a large set of microspheres is hardly to be reflected.

For this reason, it is proposed to describe the reduction of the number of intact microspheres $d N$ during the time $d t$ by a simple ratio, in which $\lambda$ is a constant: 


$$
d N=-\lambda N d t
$$

After integration, we obtain the kinetics of the decrease in the number of all microspheres with time:

$$
N=N_{0} \exp (-\lambda t)
$$

Let us consider the two mentionedabove and the most acceptable modes of suspension loading.

Changing the volume of the capsule while the microspheres remain intact:

$$
\Delta V=\pi b^{2} L\left[n 6 \frac{p}{E}(1-\mu) \frac{R^{4}}{\delta}+p\left(1-n \frac{4}{3} \pi R^{3}\right) \beta_{\text {water }}\right]=\pi b^{2} \Delta L
$$

Let us consider the case when all the microspheres collapsed. Then the volume of glass is determined by the formula:

$$
V_{k} n \frac{4}{3} \pi R^{3} 0,105 \approx V_{g l}=N \frac{4}{3} \pi R^{3} 0,105
$$

The volume of water remained almost the same: $V_{\text {water }}=V_{0}-N \frac{4}{3} \pi R^{3}$.

The relative change in capsule volume to the original:

$$
\frac{V_{0}-\left(V_{g l}+V_{\text {water }}\right)}{V_{0}}=\frac{V_{0}-V_{0}+0,895 N \frac{4}{3} \pi R^{3}}{V_{0}}=0,895 v_{s p h},
$$

where $v_{s p h}$ - relative initial volume of microspheres.

For example, at $v_{s p h}=0.5$ the volume of the capsule will be 0.5525 after the destruction of microspheres from the initial volume $V_{0}$, i.e. it will decrease almost twice. At $v_{s p h}=0.7$ the new capsule volume will decrease by more than two and a half times $\mathrm{V}_{l}=0.3735 V_{0}$. Such changes cannot be neglected. Therefore, after the destruction of all microspheres, it is necessary to follow the change in this capsule volume composed of water and glass fragments.

\subsubsection{Constant pressure (power) growth rate mode}

In this case, the destruction is determined only by the value of the pressure. The task is to determine the interval of pressures that destroy the glass microspheres. For this reason, we begin with the mode of pressure growth at a constant predetermined rate. Let us suppose that the conditional start of destruction at $p=p_{c r}$, when $1 \%$ of the spheres collapsed, and the conditional ending at $p=p_{\max }$, when $99 \%$ of the spheres collapsed. Then from (7) we have:

$$
N=N_{01} \exp (-\lambda t)=N_{01} \exp \left(-\lambda \frac{p-p_{c r}}{q}\right)
$$

Here is $\mathrm{N}_{01}=0.99 \mathrm{~N}_{0}$. The change of volume with a change of pressure to $p_{c r}$, i.e. while the spheres remain intact, is a linear function of pressure:

$$
\Delta V=\pi b^{2} L p\left[\frac{6 n}{E}(1-\mu) \frac{R^{4}}{\delta}-\left(1-n \frac{4}{3} \pi R^{3}\right) \beta_{\text {water }}\right]
$$

Volume change in the destruction of microspheres: 


$$
\begin{aligned}
& \Delta V=\left[N_{01}-N(p)\right] 0,985 \frac{4}{3} \pi R^{3}=1,333 \pi R^{3} N_{01}[1-\exp (-\lambda t)]= \\
& =1,333 \pi R^{3} N_{01}\left[1-\exp \left(-\lambda \frac{p-p_{c r}}{q}\right)\right]
\end{aligned}
$$

At the end of the destruction of almost all microspheres, the new initial volume of the capsule will be composed of the volume of glass from the destroyed microspheres $V_{g l}$ from (13) and the constant volume of water.

The change in this volume is described by the formula:

$$
\Delta V_{1}=p\left[\left(V_{0}-N \frac{4}{3} \pi R^{3}\right) \beta_{\text {water }}+0,105 N \frac{4}{3} \pi R^{3} \beta_{g l}\right]
$$

The obtained formulae for the volume change $\Delta V$ demonstrate, that the initial stage of tests (before the destruction of glass microspheres (12)) and the final stage of tests (after the destruction of almost all glass microspheres (14)) are linear functions of pressure. It can be seen from these formulae that the angle of the straight line at the first stage before the destruction is greater than the angle of the straight line at the stage after the destruction. The fracture stage (reduction of the number of intact microspheres) is presented in formulae (11) and (13) by nonlinear (exponential) function of pressure (or time) at a relatively short pressure interval $p_{c r} \leq p \leq p_{\max }$. The function starts on the first straight line, ends on the second straight line, and determines the largest decrease in volume. This section is most clearly reflected in the diagram $\Delta V(p)$ (Fig. 6). Also this section allows us to assess the average strength of glass microspheres and its dispersion. The width of this sectionreflects the real dispersion due to the geometry of the glass spheres.

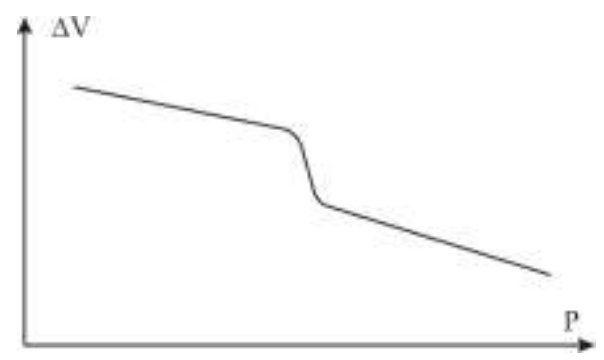

Fig. 6. The change in the suspension volume from the pressure at a constant rate of pressure growth with time.

\subsubsection{Compression with a constant rate of decrease in the volume of the suspension (constant speed of the movement of the piston)}

In these tests, we monitor the pressure. In the first and third stages of these tests, formulae (12) and (14) are valid. They reflect the linear relationship between the change in the volume of the suspension and the pressure. Formula (13) reflects an essentially nonlinear relationship between volume change and pressure. It shows that the number of destroyed glass microspheres in the first test mode changes exponentially with pressure. In the second mode, their number depends on the pressure logarithmically (much slower).

Let us suppose that the whole set of glass spheres is divided into four narrow fractions (comparable in number of microspheres) by strength. It follows that each fraction is destroyed completely after reaching a certain pressure $p_{4}>p_{3}>p_{2}>p_{1}$. Let us suppose that the rate of 
change in volume is small. While the microspheres are intact, the pressure changes linearly with the growth of the volume change $|\Delta V|$ (i.e., the volume reduction).

When the first critical pressure $p_{1}$ is reached, the first fraction of the glass spheres collapses very quickly. After that, the pressure drops sharply. As the piston continues to move at a constant slow speed, the pressure begins to rises lowly to the second critical pressure $p_{2}>p_{1}$. When the pressure reaches the second critical value $p_{2}$, a sharp drop follows again. Then the pressure slowly increases to the third critical value $p_{3}>p_{2}$.At the fourth stage, everything will repeat. In this case, between the two straight lines (the beginning and the end of the destruction of the glass microspheres), four stretched sections are obtained. Each of these sections reflects the strength of the corresponding fraction. If there are many fractions (or there is a continuous distribution of the strength of the glass microspheres), then the jumps are small. In this case, we obtain a smooth curve passing in a wide range of volume changes from straight line 1 to line 2, as in our experiment in Fig. 2.

\section{Conclusion}

The main goal of this paper is to determine the signs and criteria of destruction of the spheroplastics (syntactic foam) under hydrostatic compression. Signs of the destruction were detected through the use of the colored liquid that penetrated actively into the destructed area of the material. The destruction of the cylindrical sample of the syntactic foam under uniform compression is inhomogeneous along of the radius. Basically the outer layers of the samples are destroyed. Only at a high volume content of the glass microspheres, the entire volume of the sample is destroyed. In this case, the separation of the samples into separate parts is not observed. The fracture stages of the samples with using a constant rate of decrease in the volume are characterized by a slight change of the curvature on the test diagrams (Fig. 2). The reasons for this stretching are in the distribution of the geometric parameters of the glass microspheres and in the test method. Similar processes are observed in the compression of the suspension of the glass spheres in water.

Mathematical modeling and experiment with using a constant rate of decrease in the volume demonstrate that the stages of fracture on the pressure-volume dependence curves (the number of destroyed glass microspheres) are characterized by a slight changes of the curvature (according to the logarithmic law (13)). In tests with a constant rate of the pressure growth, the diagram of the dependence of the volume on pressure shows jumps. These jumps demonstrate the destruction of the glass microspheres (exponential fall of the volume in a narrow range of pressure change (13)). This fact allows us to assume that tests of syntactic foam in a constant rate of pressure growth allow more accurate evaluation of the strength of syntactic composites.

This work was done on the Theme No. 45.11 of the State assignment of the Federal Agency for Scientific Organizations (FASO Russia) (the Theme of FASO Russia number: 0082-2014-0009, State registration number: AAAA-A17-117040610309-0).

\section{References}

1. A.M. Kuperman, R.A. Turusov, A.J. Gorenberg, V.I. Solodilov, R.A. Korokhin, Y.A. Gorbatkina, A.V. Baikov, Mech. of Compos. Mater. 50(6), 705-716 (2015)

2. A.V. Baikov, R.A. Korokhin, V.I. Solodilov, A.Ya. Gorenberg, V.G. IvanovaMumjieva, U.G. Zvereva, A.M. Kuperman, Compos. And Nanostruct. 9(1), 1-11 (2017)

3. M. Le Gall, D. Choqueuse, P.-Y. Le Gac, P. Davies, D. Perreux, Polymer Testing 39, 36-44 (2014) 
4. J. Lachambre, E. Maire, J. Adrien, D. Choqueuse, Acta Materialia 61(11), 4035-4043 (2013)

5. F. Grosjean, N. Bouchonneau, D. Choqueuse, V. Sauvant-Moynot, J. of Mater. Sci. 44(6), 1462-1468 (2009)

6. R. Huang, P. Li, Compos. Part B: Eng. 78, 401-408 (2015)

7. P. Li, N. Petrinic, C.R. Siviour, R. Froud, J.M. Reed, Mater. Sci. and Eng. A 515(1-2), 19-25 (2009).

8. C. Swetha, R. Kumar, Mater. \& Design 32(8-9), 4152-4163 (2011)

9. E.M. Wouterson, F.Y.C. Boey, X. Hu, S.-C. Wong, Compos. Sci. and Tech. 65(11-12), $1840-1850(2005)$ 